Pragmatic Case Studies in Psychotherapy, http://pcsp.libraries.rutgers.edu

Volume 13, Module 1, Article 2, pp. 38-50, 04-22-17 [copyright by author]

\title{
Commentary on The Case of "Hiro": Treating Tourette Syndrome by Comprehensive Behavioral Intervention for Tics (CBIT)
}

\section{On Protocols and Principles in the Case of Hiro}

\section{MATTHEW R. CAPRIOTTI ${ }^{\mathrm{a}, \mathrm{b}}$}

\author{
a San José State University, San José, CA \\ ${ }^{\mathrm{b}}$ Correspondence regarding this article should be sent to Matthew R. Capriotti, San José State University, \\ Department of Psychology, One Washington Square, San José, CA 95192-0120 \\ Email: matthew.capriotti@sjsu.edu
}

\begin{abstract}
Dr. Jeremy Lichtman (2017, in this issue) presents a case of successful behavioral treatment of tics in "Hiro," a 10-year-old boy with Tourette Syndrome (TS) and attention-deficit/hyperactivity disorder (ADHD). This commentary discusses the case of Hiro from a principle-oriented approach, with particular focus on issues related to parental involvement in treatment, addressing comorbidity and therapy-interfering behavior, and planning for generalization and long-term maintenance.

Key words: Tourette Syndrome; Tics Disorders; Comprehensive Behavioral Intervention for Tics (CBIT); Habit Reversal Training (HRT); cognitive-behavioral training (CBT); protocol-based manualized therapy; principles-based manualized therapy; parent involvement; comorbidity; therapy-interfering behavior; case studies; clinical case studies
\end{abstract}

Effective behavioral treatments for tic disorders have existed for decades. Early reports (e.g., Azrin \& Nunn, 1973) focused on the use of habit-reversal training (HRT), a multicomponent treatment package designed to decrease an array of body-focused repetitive behaviors, including tics, hair pulling, and skin picking. These treatments, rooted in a behavioranalytic view of tics (and human behavior in general) showed large and durable treatment effects. In the past decade, behavior therapy for tics has "gone mainstream," with the development, testing, and dissemination of Comprehensive Behavioral Intervention for Tics (CBIT; Woods et al., 2008). CBIT is a contemporary treatment program that incorporates early behavioral approaches (e.g., HRT) with other behavior therapy techniques. Recent multisite randomized controlled trials have demonstrated that CBIT is efficacious in both youth (Piacentini et al., 2010) and adults (Wilhelm et al., 2012), with treatment effects comparable to psychotropic medications, but without significant side effects (Scahill et al., 2013). In light of this strong efficacy data, CBIT has been disseminated through various avenues. The CBIT manual (Woods et al., 2008), which is written in a style accessible to a variety of mental health professionals is available across the globe in various languages. In the U.S., the Tourette Association of America (TAA; https://www.tourette.org/) sponsors a formal Behavior Therapy 
Pragmatic Case Studies in Psychotherapy, http://pcsp.libraries.rutgers.edu

Volume 13, Module 1, Article 2, pp. 38-50, 04-22-17 [copyright by author]

Institute to provide high-quality CBIT training to professionals (TAA, 2017; https://www.tourette.org/blogs/research-medical/cbit-bti-training/ ). Modified forms of CBIT designed to be transported to neurology and occupational therapy clinics have been developed and show promise (Ricketts et al., 2016; Rowe, Yuen, \& Dure, 2013).

\section{THE ROLE OF TREATMENT MANUALS IN EVIDENCE-BASED PRACTICE}

Evidence-based practice (EBP) of psychotherapy tasks clinicians with blending best available research evidence, clinical expertise, and knowledge of client characteristics to inform their delivery of psychological services (Anderson, 2006). Not only do clinicians practicing EBP "blend art and science" to plan treatment, but they also rely on ongoing progress monitor to determine if the treatment is having the expected clinical benefit in the case at hand (Anderson, 2006; Patterson et al., 2004). The CBIT manual, like many treatment manuals, provides a prescriptive session-by-session approach, accompanied by general instructions for the clinician to use their clinical judgement in adapting the manual to their individual clients' needs. This need for clinicians to exercise "flexibility within fidelity" in their use of manualized treatments has received growing attention in the research literature (e.g., Breitenstein et al., 2010; Hamilton, Kendall, Gosch, Furr, \& Sood, 2008). In fact, this increased attention to the need for creativity and individualization of treatment manuals has even led some manual authors to explicitly flag have opportunities for individualization within the session-by-session protocols (e.g., "Tips From the Trenches” in the latest $\left(3^{\text {rd }}\right)$ edition of the Coping Cat treatment for child anxiety; Kendall \& Hedtke, 2006).

Given that the child psychotherapy research literature is largely built on studies that have employed manualized treatments, it is worth considering more specifically how these might be integrated into EBP. In my own training and clinical practice, I have been exposed to two general approaches to incorporating treatment manuals into EBPs. Though I have heard many clinicians talk about these issues, I have not seen them clearly defined and discussed in the research literature, so I will attempt to do so briefly here. My discussion of these approaches here is tentative, relatively informal, and meant to serve as a heuristic for further discussion herein; these ideas are not intended as formal iterations of program theories, logic models, etc. about implementing empirically supported treatments. Also, my general view is also not that one approach is inherently superior; there are pros and cons to both. In fact, I think a well-informed clinician may find themselves taking one approach in some situations and the other at different times.

The first approach might be called "Protocol First." In this approach, a therapist selects a treatment protocol for treating a client who is diagnostically and demographically similar to participants in a clinical trial that found the protocol efficacious. That is, the protocol is deemed appropriate if it has shown efficacy for similar clients in clinical trials. Once an appropriate protocol is identified, the treatment is delivered as manualized. If treatment proceeds well, the therapist keeps following the manual on a session-by-session basis. If treatment begins to "stall" (e.g., progress as measured by assessment tools has plateaued, or barriers to treatment adherence present themselves), then the therapist uses strategies outside of the manual based on their clinical judgement (i.e., conceptualization of what is going wrong and what might help). Much of 
Pragmatic Case Studies in Psychotherapy, http://pcsp.libraries.rutgers.edu

Volume 13, Module 1, Article 2, pp. 38-50, 04-22-17 [copyright by author]

the rationale for this view is that empirically-supported treatments have demonstrated efficacy when done in a certain, very particular order (i.e., as manualized). Thus, the more the therapist deviates from the manual, the less confident they can be that the (successful) results of the efficacy trial will generalize to their therapy with this client. That said, in this view, when treatment is clearly at risk of failing, the therapist has good reason to put the manual aside and use their clinical judgment to achieve progress.

The second approach, which I will call "Principles First,” generally holds that the clinical power of empirically-validated treatment protocols derives from their use of cognitive-behavioral principles. In this view, empirically-validated manual is seen as an exemplar of one way in which procedures might be meaningfully synthesized into a coherent treatment for an individual client with a specific presenting problem. The manual may then be used as a tentative framework for delivering treatment, and the clinician knows that treatment based on this treatment manual has shown to be more effective than other treatments (i.e., the treatment is efficacious). Critically, in this approach, the therapist may anticipate that the manualized treatment will need modification before therapy actually begins. So, the initial treatment plan is then formed by integrating the therapist's idiographic case conceptualization and the empiricallyvalidated protocol. If the clinician's case formulation seems to suggest that treatment elements from the manual be omitted, re-ordered, expanded, contracted or otherwise altered, and/or that adding additional elements could be useful, then they are encouraged to do so. Just as in a "Protocol First" approach, ongoing outcome monitoring is used to determine if the current treatment plan is viable, or if corrections are needed.

In my view, the case of Hiro by Dr. Jeremy Lichman (2017, this issue) is a clear and clinically successful application of the Protocol First approach. The therapist begins with a plan to follow an empirically-tested treatment protocol. Then, he uses both quantitative and qualitative data to determine that treatment is not working; next, he changes his intervention approach to address challenges that have presented. Ultimately, good treatment progress is obtained through this revised approach. This process clearly exemplifies evidence-based practice. Here, I will consider how a Principles First approach may have led to different perspectives on this case and perhaps suggested other possibilities for clinical action. My discussion will focus on three content-related themes: a) involvement of parents in therapy, b) a function-analytic view of nonadherence, and c) programming for generalization and maintenance. These topics are indeed common issues in the "real-world" clinical practice of treating tic disorders, and my discussion here will tackle them from a bottom-up, principledriven analysis of this case. I hope these will serve to give additional perspectives on the second and third themes that Dr. Lichtman proposes in the case of Hiro: "How does ADHD impact CBIT treatment success and what can be done about it?" and "How can a clinician respond to challenging elements in a TS case?”

Before diving into commentary, I want to be clear about my central thesis. In reading this case, it is clear to me that Hiro's clinical endpoint is quite favorable and Dr. Lichtman's clinical approach soundly abides by tried-and-true principles of EBP. My main hunch is that a Principles First approach could have arrived at a similar clinical endpoint as the Protocol First approach demonstrated in this case (a fine example of multifinality, which Dr. Lichtman (2017) refers to in his introduction (p. 9). However, I do wonder if a Principles First approach might have made this 
Pragmatic Case Studies in Psychotherapy, http://pcsp.libraries.rutgers.edu

Volume 13, Module 1, Article 2, pp. 38-50, 04-22-17 [copyright by author]

therapy more efficient by reducing both the amount of therapy needed and the degree of frustration experienced by the child, family, and therapist. Essentially, my suggestion here is that a Principles First approach might have made a very good course of therapy even better in a couple of important ways.

\section{PARENT INVOLVEMENT}

The CBIT manual, designed to guide treatment for child, adolescent, and adult patients with tics, advises that parents be significantly involved in the assessment and treatment process, but does not make explicit prescriptions about the each specific way this might occur. One CBIT component that concretely pulls for parental involvement is the "social support" component of HRT, in which a "support person" (e.g., parent, other caregiver, spouse, roommate) is trained: (a) to praise the client's use of tic-management skills, and (b) to prompt the client to use these skills when the client is not doing so independently. With minor clients, the parent(s) will nearly always serve this role, and the CBIT manual is quite clear on this point. However, aside from this "core requirement," the involvement of parents in CBIT depends on the clinician's expertise and perspective on this clients individual characteristics (e.g., view of contextual and developmental factors at play). Thus, the clinician has considerable latitude here in deciding the degree to which parents are involved in therapy sessions. Clinicians' thinking around this may be influenced by a litany of factors including the child's level of independence, parent-child relationship dynamics, and other factors. That said, age alone will often provide a good starting point for thinking about degree of parent involvement. For most 10 year olds undergoing behavioral and cognitivebehavioral interventions, it is common practice to involve parents in significant parts of each session.

In the case of Hiro, it seems as though the client's parents were involved in some parts of early sessions (e.g., psychoeducation), though it is unclear if and how they participated in HRT and its component parts (awareness training, competing response training, and social support). Working backward from Dr. Lichtman's description of Sessions 14 and 15, it seems as though parental involvement in HRT components of sessions was minimal. For example, Dr. Lichtman writes that the plan for Session 14 was for "Hiro's parents to observe me going through a typical session with Hiro, with me pausing along the way to explain what we were doing and why was" (2-17, p. 22); and that in Session 15, "Hiro was asked then about what it was like having parents in the session” (2017, p. 22), implying that this had not happened in earlier HRT sessions. As Dr. Lichtman notes, Hiro's parents' involvement appeared integral to the ultimate success of treatment. This fits with both my own clinical experience, and my read of the child behavior therapy research literature (both broadly and with regard to TS and related disorders). Ultimately, after beginning with relatively little involvement, the therapist then increased the degree of parental involvement in sessions, which seems to have helped produce a good clinical outcome. In the case of Hiro, I think earlier and more active involvement of his parents could have been used as part of a bottom-up approach to three core complications that arose in therapy: homework adherence, parent-child interactions around therapy tasks, and parental buy-in/motivation.

First, involving parents more actively in early sessions may have helped prevent and/or more quickly address problems with homework adherence. Hiro’s struggles with homework 
Pragmatic Case Studies in Psychotherapy, http://pcsp.libraries.rutgers.edu

Volume 13, Module 1, Article 2, pp. 38-50, 04-22-17 [copyright by author]

adherence were noted fairly early in treatment; during Session 4, his mother reports he "was not practicing at home.” It was not until Session 7 that Hiro returned a fully completed homework form. The CBIT manual prescribes two main strategies to support homework compliance, and both of these can be enhanced with active parental involvement. The first component is a behavioral reward plan to target homework adherence from the beginning of treatment. Ideally, the therapist, parent, and child spend a portion of the first therapy session collaboratively generating an age-appropriate reward plan to target session attendance, in-session participation, and homework completion. Parental involvement in this process can help boost the child's motivation for the reward (and the therapy in general), specifically when parents express enthusiasm for the child earning the reward. It also makes clear that the parent, therapist, and child are "on the same page" regarding expectations for therapy participation.

The CBIT manual also prescribes a brief discussion of homework assigned for the upcoming week at the end of the session. When parents are present for this, they often prompt a discussion of when, where, and how homework might be completed. For instance, if a therapist assigns a child to practice a competing response while self-monitoring their tics four times for 30 minutes each, a parent might mention that the child is going to be camping with friends that weekend, and therefore will have to practice on the weeknights before and after the camping trip. Thus, when parents are involved in session when homework is assigned, there is maximal time for planning homework adherence in the face of logistical, scheduling and other kinds of barriers. Additionally, for a client this age, parents might be tasked with providing reminders and scaffolding for children to complete therapy homework; in other words, it is often reasonable to charge parents with "remembering to do the homework" and the child merely with complying with the parents' prompt to do the homework. Of course, this depends on the parents' having a solid understanding of treatment skills and activities, which is best accomplished with significant involvement in each session.

Second, involving parents extensively in early therapy sessions allows the therapist to make important observations about parent-child dynamics, both broadly and as they relate directly to the current therapy. I will not speculate broadly on Hiro’s global family dynamics here, as there is little information in the case discussion to draw on in this area. That said, given the parents' frustration with early-stage treatment, combined with the low homework compliance, it seems safe to say that parent-child interactions around practicing the tic exercises were likely not optimally effective. By involving parents in sessions, clinicians can observe important samples of parent, child, and dyadic behaviors. Some of these relate to treatment knowledge and parent skill acquisition: Does the parent reflect correct understanding of when and how the child is supposed to use competing responses? Is the parent able to notice when the child is using the competing response in session, and, subsequently, provide praise to the child?

On another note, the therapist can observe salient features of parent-child interactions around tics in session: Does the child "shut down" or become withdrawn when the parent discusses their tics or prompts them to use the competing response? When the parent prompts for competing response use, what is their tone like? By observing issues like these during sessions, the clinician may gain an idea of dyadic factors that facilitate and/or complicate effective parentchild teamwork around tics, and in general. These observations can provide important qualitative assessment data to inform later problem solving in parent-child interactions related to therapy 
Pragmatic Case Studies in Psychotherapy, http://pcsp.libraries.rutgers.edu

Volume 13, Module 1, Article 2, pp. 38-50, 04-22-17 [copyright by author]

(e.g., completing homework, serving as a support person for competing response use). One interesting point is the observation in the final treatment session, when Hiro's parents make an off-handed comment that they don't "get the kid stuff," (Lichtman, 2017, p. 23), such as talking with Hiro about videogames. Had the parents been involved heavily in early sessions, I wonder if the therapist might have observed their difficulties engaging in conversation around topics of interest to Hiro. If this occurred, it may have occasioned the therapist to work on these skills with the parents. For instance, the therapist might have explicitly worked with the parents and child on the skills of how to have a conversation about "kid stuff" together. This might have facilitated higher quality conversations not only when working on tic-related skills, but also at other times, which may have led to a global improvement in Hiro's relationships with his parents. While admittedly speculative, these kinds of processes could account, in part, for decreases in family strain observed following successful CBIT (Woods et al., 2011).

Third, involving parents throughout the course of the treatment process can increase their own motivation and buy-in. Dr. Lichtman suspected problems in these domains in the early stages of Hiro's treatment. When parents are involved in in-session activities that are aimed at increasing the child's motivation, their motivation to facilitate their child's active participation will likely increase too. For instance, during Session 2 of the CBIT manual, clients complete a list of Tic Hassles (i.e., specific areas of tic-related impairment) that are then tracked across treatment. When parents participate in making this list, they are reminded of the tic-related challenges that their child faces, and they may see these as problems that therapy is specifically designed to address. This process can function to increase the parent's motivation when they are involved in this conversation. Parents then may have the chance to watch as the child and therapist update this list toward the beginning of Sessions 3-8. When therapy is succeeding, children generally report decreased tic severity and less impairment from hassles. The therapist and client are often genuinely quite excited when decreases in hassles are observed, and this happy event can function as a motivator for continuing to use therapy skills (e.g., practicing competing responses). When parents are present for this part of the session, it also creates an opportunity to directly praise and enumerate things that the parents are doing to effectively support the child's progress in therapy. Of course, other aspects of participating actively in therapy sessions can also boost parental motivation.

\section{AN ALTERNATIVE, PARENT-FOCUSED VIEW}

When considering idiographic approaches to case conceptualization, it is common to consider alternative formulations and/or approaches to treatment in one's clinical thinking. This is not intended as a mere academic exercise, nor is it designed to induce self-doubt for its own sake. Rather, this activity can lead to appropriate critical thinking about the use of one's primary formulation or initially preferred approach. This practice may also lead one to seriously consider adopting alternative courses of treatment, without requiring abandonment of the epistemic foundations of the therapist's outlook.

For these purposes, I consider here an alternative therapy course in which an entirely parent-focused approach could have been used. Considering the information reported from the intake phase, treatment was initiated by the parents and did not appear to be motivated by current impairment or child distress related to tics, as Hiro "entered treatment at this time because his 
Pragmatic Case Studies in Psychotherapy, http://pcsp.libraries.rutgers.edu

Volume 13, Module 1, Article 2, pp. 38-50, 04-22-17 [copyright by author]

parents were worried that his tics would continue to worsen and impede his future ability to succeed” (Lichtman, 2017, p. 12). Consistent with this view, Hiro was experiencing only "minimal" current impairment due to tics (10/50 on the YGTSS Impairment Scale). Though the nature of this impairment is not directly discussed in Hiro's case study, this omission seems to argue the view that Hiro was not bothered much by his tics at treatment onset. In contrast, it does seem that his parents were distressed about them. Given this presentation, one might have reasoned that Hiro's "intrinsic” motivation to use tic-management skills might have been low (given the absence of tic-related impairment).

Based on this understanding, the therapist might have focused on working with the parents on psychoeducation and parent-training components of CBIT. Psychoeducation often has the effect of decreasing parental distress about their child's behavioral/emotional problems. For this case, Hiro's parents' primary stated motivation for seeking treatment for him was a worry that the symptoms might worsen over time. Thus, they might have been quite comforted to know that most children with tics experience their maximum tic severity around their son's age (10-12 years; Bloch \& Leckman, 2009), and that tics tend to decrease in severity throughout older adolescence and young adulthood. Additionally, it seems that they would have benefitted from learning about environmental influences on tics, and the notion of creating a "tic neutral environment," in which planned routines are designed to minimize inadvertently reinforcing responses to tics (e.g., providing comfort when a child is ticcing, taking a break from homework when a "burst" of tics occurs). These treatment elements correspond to the "Functional Assessment and Intervention” component of the CBIT manual (Woods et al., 2008), and do not necessarily depend on the child's participation. These elements of treatment alone can help to alleviate parental anxiety about the child's future experience of tics. They also promote a degree of self-efficacy by showing parents that they can do something active to help with the tics (i.e., implementing the strategies suggested in session for reacting to tics in a neutral way and effectively coaching their child around coping with tics). If further assessment indeed revealed that Hiro was not substantially bothered by his tics, these treatment components alone may have been sufficient to resolve the tic-related issues that were problematic in Hiro's case.

In considering the above alternative, I notice the importance of the parents' view of their child's tics. Parental distress around Hiro's tics was a primary motivator of them coming to therapy, so it makes sense to address this issue directly in treatment. By the end of therapy, it seems that Hiro's parents indeed were much less distressed about Hiro's tics and his future. So, the actual clinical approach that Dr. Lichtman took achieved this goal, just as the alternative parents-only approach might have. Also, the importance of building Hiro's motivation and planning for child adherence comes to the forefront. The above alternative can be a feasible course of action when parents are distressed by a child's tics while the child is not. However, the approach that Dr. Lichtman actually employed did involve Hiro learning many skills and doing many effortful tasks. In the next section, I discuss perspectives on tackling challenges related to treatment adherence, which may been seen as related to low levels of "intrinsic" motivation and Hiro’s comorbid ADHD. 
Pragmatic Case Studies in Psychotherapy, http://pcsp.libraries.rutgers.edu

Volume 13, Module 1, Article 2, pp. 38-50, 04-22-17 [copyright by author]

\section{MAKING SENSE OF COMORBIDITY IN TREATMENT: A FUNCTION-ANALYTIC LENS}

Behaviorally and cognitive-behaviorally oriented clinicians are used to generating neat case conceptualizations based on tried-and-trued principles. In these formulations, the proposed mechanisms of pathology (e.g., distorted thinking, skill deficits, relative availability of reinforcement for desired vs. undesired behavior) lead the clinician to clear ideas about interventions that will fairly directly target relevant mechanisms of change (e.g., correcting thinking errors, building functional skills, creating environmental modifications to alter the availability of reinforcement for desired behavior). This straightforward mapping of intervention to mechanism is often touted as a strength of cognitive and behavioral therapies. However, straightforward behavioral conceptualizations are quickly complicated by the complexity of our clients' presentations and contexts.

In behavior therapy for TS, one common aspect of "complication” involves comorbidity. Many, if not most, children with TS meet criteria for another DSM diagnosis, with attentiondeficit/hyperactivity disorder (ADHD) and obsessive-compulsive disorder (OCD) being the most common co-occurring conditions (Kadesjo \& Gillberg, 2000). Many research studies have detailed this overlap, and many well-controlled studies have identified underlying neural, cognitive, and behavioral processes that might contribute dually to TS and these comorbidities. On the clinical side, a fair amount has been written about considering comorbidity in TS, but direct recommendations remain fairly general (e.g., treat the most impairing problem first, consider comorbidity when selecting pharmacological agents, and comorbidity may complicate treatment).

At present, there are not specific, empirically-supported answers to core clinical questions, such as, “[how] do I modify CBIT for youth with ADHD?” Though indirect metaanalytic evidence suggests that ADHD may influence CBIT outcomes (Maguire et al., 2014), the child CBIT trial found efficacy in a sample where 26\% of participants had ADHD (Specht et al., 2011. Experimental findings from laboratory studies (e.g., Lyon et al., 2010) also suggest that children with ADHD are able to inhibit their tics just as well as those without ADHD. Given this picture, it is clear that: (a) clinicians can and should provide behavior therapy for tics with kids who have ADHD and other co-occurring problems (when tics are the primary concern), (b) comorbidity may complicate treatment in some cases, (c) issues that are not psychiatric comorbidity per se (e.g., parent-child conflict, child temperament, academic stress) can also influence the course of treatment significantly.

Difficulties with inattention and hyperactivity/impulsivity associated with ADHD can complicate CBIT. For Hiro, ADHD-associated difficulties may be seen as contributing to adherence problems in therapy. To address these issues, I will demonstrate how a functionanalytic view of therapy non-adherence can be applied to the adherence issues seen in Hiro's case. Specifically, I will borrow the term "therapy-interfering behavior" (TIB) from Dialectical Behavior Therapy (e.g., Linehan, 1993), though many others have considered the functional concepts at hand. In this view, the core behavioral conceptualization outlined by Dr. Lichtman's introduction is retained, and the specific therapy plan being "interfered with" is a completely "by the book” delivery of the CBIT protocol with good outcomes along the way. TIB is then defined 
Pragmatic Case Studies in Psychotherapy, http://pcsp. libraries.rutgers.edu

Volume 13, Module 1, Article 2, pp. 38-50, 04-22-17 [copyright by author]

as any pattern of behavior by the client, family, and/or therapist that interferes with the effective application of treatment as planned (e.g., by decreasing homework adherence, interfering with session attendance, or interfering with successful acquisition of therapy skills in session). Then, TIB is submitted to a functional assessment, which forms the basis for a plan designed to ameliorate the TIB in the future. As such, "barriers to treatment” are viewed and addressed in the same framework as the treatment itself. In Hiro's case, at least two types of TIB appear: low rates of completion of homework assignments and tardy arrival to sessions.

With regard to homework task completion, it is not clear exactly whose behavior was most "interfering," but Dr. Lichtman's narrative suggests that homework compliance was consistently below target level for the first five weeks in which homework was assigned. When the therapist noted the insufficient homework completion from the past week (presumably at the beginning of session during homework review), they might have begun a functional assessment of this nonadherence by asking Hiro and his parents about factors that "got in the way" of the homework complettion that week. It also may have revealed instances where Hiro's noncompletion of homework was reinforced. Of course, uncovering this kind of behavioral chain surrounding homework non-completion would require active parental involvement in the course of the session, as I've discussed above. Parental responses during this assessment process could suggest a variety of barriers to homework completion. For instance, perhaps Hiro and his parents both "forgot about" the assigned homework, and Hiro's noncompletion of the tasks simply went unnoticed until session. Perhaps his parents did prompt him to practice, Hiro declined, and the parents "backed off" of this request. Or, perhaps they got into an argument about Hiro doing the therapy homework and then disciplined Hiro with some sort of punishment, but not one that required him to do the assigned therapy skill practice. Perhaps it was a combination of these and/or other factors.

Once the therapist has a sufficient picture of the antecedents and consequences surrounding homework non-completion, they would then draw on this understanding to formulate a plan for the next week to address these factors, thereby decreasing the probability of the TIB persisting. In this process, TIB cannot simply be "explained as" comorbidity (e.g., "His ADHD just makes it too hard for him to remember to practice.”). Rather, immediately relevant behavioral and cognitive sequalae of ADHD must be identified and integrated into a specific and testable hypothesis about why non-adherence is occurring. Indeed, the comorbid diagnostic label may be helpful in considering factors contributing to the TIB (e.g., that Hiro is likely to need greater scaffolding and more immediate reward because he has ADHD), but it is not itself considered as the barrier to therapy.

Ultimately, Dr. Lichtman wound up having success with a "function-blind” intervention, in which he forwent this kind of chain analysis and used a structured behavioral reward plan to increase adherence to homework assignments. This approach was quite effective in increasing Hiro's homework adherence, and the implementation of this tool appeared to mark a "turning point" in the course of treatment. That said, a TIB frame, or "function-analytic" approach, might have led to a discussion of homework adherence earlier in the course of treatment (as soon as Session 2), which could have allowed for greater efficiency. 
Pragmatic Case Studies in Psychotherapy, http://pcsp.libraries.rutgers.edu

Volume 13, Module 1, Article 2, pp. 38-50, 04-22-17 [copyright by author]

I do think a function-analytic view of TIB would have changed Dr. Lichtman's thinking about his client's tardiness to session. The author interpreted the family's tardiness as indicative of their lack of hope in treatment. It is unclear if there were additional indicators of this, though I have noticed that it is not uncommon for many therapists (including highly competent ones) to jump to interpretations about reasons for client's non-attendance. When our clients are tardy to sessions (or don't arrive at all) this can feel quite frustrating and even personal; given this, I believe an attempt at a more detached, function-analytic account is even more important.

Viewing tardiness as TIB might have confirmed Dr. Lichtman's suspicion about motivation as a contributing factor, and/or it might have led to the discovery of other factors that were contributing to late arrivals. Perhaps Hiro's family frequently found themselves delayed as they left the house. Perhaps Hiro was adamant about not attending sessions, and a power-struggle ensued when they were about to leave. Perhaps Hiro’s parents simply forgot about the sessions until shortly beforehand. Discovering any facts like this can be helpful not only in addressing TIB, but also in identifying problematic behavioral patterns that might cause problems in non-tic areas. For instance, if this discussion revealed that the family is chronically running late to appointments as a family, this would be useful to know, as it may cause distress and impairment in non-tic-related domains as well.

Further, this information might lead to related clinical hypotheses about other targets (e.g., that the family struggles with implementing routines and planning), which might also contribute to the aforementioned issues with therapy homework compliance. On the other hand, such an analysis might reveal that the family's late arrivals were due to non-systematic factors (e.g., getting stuck behind an accident on the drive to the clinic); knowing this could have provided an opportunity for the therapist to empathize with his family's plight, build rapport with the parents, and avoid potentially unfounded negative interpretations about the reasons for their arrival time.

\section{PLANNING FOR TREATMENT GENERALIZATION AND MAINTENANCE}

Planning for beyond the end of therapy is a critical part of psychotherapeutic intervention. In CBIT, explicit planning for post-treatment success is carried out using three specific strategies: 1) providing prescriptive information about “dos and don'ts” for managing tics when they periodically increase in the natural course of TS, 2) ensuring that the client and family can accurately apply HRT skills to new tics, and 3) conducting periodic booster sessions to provide problem-solving as needed. Hiro's case added an interesting contextual wrinkle to this process: the family planned to move to India at the end of treatment. Dr. Lichtman did a commendable job of planning for the future during the last stage of treatment. I will now analyze how he used manualized and non-manualized approaches to serve each of these three aims well.

First, in the final therapy session, Dr. Lichtman notes that he and Hiro's family discussed the course of TS, along with how Hiro could use competing responses in the future to address new tics. This seems to roughly correspond to the "Relapse Prevention" component of Session 8 in the CBIT manual. Though little detail is provided on this session in the manuscript, this treatment component presumably also gave Hiro and his parents the chance to ask the therapist 
questions they had about dealing with tics in the future and in applying the strategies learned in therapy.

Dr. Lichtman did a nice job to ensure that the client and family could apply habit reversal training (HRT) to new tics (i.e., those not yet targeted in treatment). The CBIT manual indicates that children should be prompted to generate competing responses for new tics during Session 8 (Relapse Prevention), but the approach described here takes an interesting additional step: the therapist used a parent-training approach to "transfer" the therapist role to Hiro's parents for the final tics. By doing this, the therapist was able to confirm not only that the client and family knew how to describe the HRT process, but that they actually had the skills to apply HRT to a new tic with fidelity. This progression, in which the therapist first applies interventions and then later teaches parents to use them with their children, is a hallmark of many of our most powerful child behavior therapy interventions (e.g., behavioral parent training for disruptive behavior) and seemed to be a good fit for this case. By walking the family though this activity in the end stages of treatment, the clinician verifies that the family can demonstrate the skills needed to work on future tics, and the family also may become empowered by knowing that they indeed can generate effective tools for dealing with tics on their own.

Finally, I was intrigued by Dr. Lichtman's use of videoconferencing technology to enable booster sessions after the family relocated to India. At least three published studies have investigated the feasibility and efficacy of doing CBIT remotely via teleconferencing (Himle et al., 2012; Ricketts, Bauer, et al., 2016; Ricketts, Goetz, et al., 2016). In these studies, CBIT has demonstrated efficacy when delivered entirely via videoconferencing. The present application is interesting, as it "transfers" what begins as in-person therapy to a remote treatment modality. Anecdotally, this approach has become rather common, especially in private practice settings, when a client relocates and wishes to keep their same therapist. Interestingly, little research (at least that I'm aware of) has investigated the effects of this transfer of modality (from face-to-face to videoconferencing) on therapeutic alliance and clinical outcomes. It certainly does seem to have many appealing aspects, and I was glad to see that it allowed Dr. Lichtman to provide booster sessions following this successful treatment case. I think readers would also benefit from "on-the-ground" perspectives from clinicians navigating issues around licensure and billing in these kinds of areas. I hope we will see more clinicians publishing on their work surrounding telehealth and transferring face-to-face therapy to videoconferencing; this is a prime example of an area where practitioners' clinical experiences can set the occasion for research on a topic of great import.

\section{CONCLUSION}

Effective evidence-based practice requires clinicians to pay careful attention both to treatment procedures they employ and the principles which they hope to harness to affect change. This is true regardless of the clinical issue at hand, but can become critical to the success of therapy when "complications arise." In working with youth with tic disorders, "complications" due to comorbidity and contextual factors can be regarded as the rule, rather than the exception. Dr. Lichtman describes an elegant, creative, and empirically-grounded approach to adapting the manualized CBIT protocol in working with Hiro, a 10-year-old client 
Pragmatic Case Studies in Psychotherapy, http://pcsp.libraries.rutgers.edu

Volume 13, Module 1, Article 2, pp. 38-50, 04-22-17 [copyright by author]

with TS. Here, I have discussed several ways in which a principle-driven view might have led to a different series of modifications at different times in the course of treatment.

My thoughts here might be seen as variations on Dr. Lichtman's theme of providing individualized, evidence-based treatment for Hiro's TS. As behavior therapy grows in popularity as a treatment for tic disorders, I hope that clinicians will riff on this theme in their own ways, by adopting thoughtful approaches to providing services to clients with tic disorders that are both appropriately individualized and thoroughly grounded in clinical research evidence and behavioral principles.

\section{REFERENCES}

Azrin, N. H., \& Nunn, R. G. (1973). Habit-reversal: a method of eliminating nervous habits and tics. Behaviour Research and Therapy, 11, 619-628.

Bloch, M. H., \& Leckman, J. F. (2009). Clinical course of Tourette syndrome. Journal of Psychosomatic Research, 67, 497-501.

Breitenstein, S. M., Gross, D., Garvey, C. A., Hill, C., Fogg, L., \& Resnick, B. (2010). Implementation fidelity in community-based interventions. Research in Nursing \& Health, 33, 164-173.

Hamilton, J. D., Kendall, P. C., Gosch, E., Furr, J. M., \& Sood, E. (2008). Flexibility within fidelity. Journal of the American Academy of Child \& Adolescent Psychiatry, 47, 987993.

Himle, M. B., Freitag, M., Walther, M., Franklin, S. A., Ely, L., \& Woods, D. W. (2012). A randomized pilot trial comparing videoconference versus face-to-face delivery of behavior therapy for childhood tic disorders. Behaviour Research and Therapy, 50, 565570.

Kadesjö, B., \& Gillberg, C. (2000). Tourette's disorder: epidemiology and comorbidity in primary school children. Journal of the American Academy of Child \& Adolescent Psychiatry, 39, 548-555.

Kendall, P. C., \& Hedtke, K. A. (2006). Cognitive-behavioral therapy for anxious youth: Therapist manual (3rd ed.). Ardmore, PA: Workbook Publishing.

Lichtman, J.D. (2017). The case of “Hiro”: Treating Tourette Syndrome by Comprehensive Behavioral Intervention for Tics (CBIT). Pragmatic Case Studies in Psychotherapy, 13(1), Article 1, 1-37. Available: http://pcsp.libraries.rutgers.edu

Linehan, M. (1993). Cognitive-behavioral treatment of borderline personality disorder. Guilford press.

Lyon, G. J., Samar, S. M., Conelea, C., Trujillo, M. R., Lipinski, C. M., Bauer, C. C., ... \& Castellanos, F. X. (2010). Testing tic suppression: Comparing the effects of dexmethylphenidate to no medication in children and adolescents with attentiondeficit/hyperactivity disorder and Tourette's disorder. Journal of Child and Adolescent Psychopharmacology, 20, 283-289.

McGuire, J. F., Piacentini, J., Brennan, E. A., Lewin, A. B., Murphy, T. K., Small, B. J., \& Storch, E. A. (2014). A meta-analysis of behavior therapy for Tourette syndrome. Journal of Psychiatric Research, 50, 106-112.

Patterson, J. E., Miller, R. B., Carnes, S., \& Wilson, S. (2004). Evidence-based practice for marriage and family therapists. Journal of Marital and Family Therapy, 30, 183-195. 
Pragmatic Case Studies in Psychotherapy, http://pcsp. libraries.rutgers.edu

Volume 13, Module 1, Article 2, pp. 38-50, 04-22-17 [copyright by author]

Piacentini, J., Woods, D. W., Scahill, L., Wilhelm, S., Peterson, A. L., Chang, S., ... \& Walkup, J. T. (2010). Behavior therapy for children with Tourette disorder: A randomized controlled trial. Journal of the American Medical Association, 303, 1929-1937.

Ricketts, E. J., Bauer, C. C., Ran, D., Himle, M. B., \& Woods, D. W. (2016). Pilot open case series of voice over internet protocol-delivered assessment and behavior therapy for chronic tic disorders. Cognitive and Behavioral Practice, 23, 40-50.

Ricketts, E. J., Gilbert, D. L., Zinner, S. H., Mink, J. W., Lipps, T. D., Wiegand, G. A., ... \& Woods, D. W. (2016). Pilot testing behavior therapy for chronic tic disorders in neurology and developmental pediatrics clinics. Journal of Child Neurology, 31, 444450.

Ricketts, E. J., Goetz, A. R., Capriotti, M. R., Bauer, C. C., Brei, N. G., Himle, M. B., ... \& Woods, D. W. (2016). A randomized waitlist-controlled pilot trial of voice over Internet protocol-delivered behavior therapy for youth with chronic tic disorders. Journal of Telemedicine and Telecare, 22, 153-162.

Rowe, J., Yuen, H. K., \& Dure, L. S. (2013). Comprehensive behavioral intervention to improve occupational performance in children with Tourette disorder. American Journal of Occupational Therapy, 67, 194-200.

Scahill, L., Woods, D. W., Himle, M. B., Peterson, A. L., Wilhelm, S., Piacentini, J. C., ... \& Mink, J. W. (2013). Current controversies on the role of behavior therapy in Tourette syndrome. Movement Disorders, 28, 1179-1183.

Specht, M. W., Woods, D. W., Piacentini, J., Scahill, L., Wilhelm, S., Peterson, A. L., ... \& Buzzella, B. A. (2011). Clinical characteristics of children and adolescents with a primary tic disorder. Journal of Developmental and Physical Disabilities, 23, $15-31$.

Wilhelm, S., Peterson, A. L., Piacentini, J., Woods, D. W., Deckersbach, T., Sukhodolsky, D. G., ... \& Scahill, L. (2012). Randomized trial of behavior therapy for adults with Tourette syndrome. Archives of General Psychiatry, 69, 795-803.

Woods, D. W., Piacentini, J., Chang, S., Deckersbach, T., Ginsburg, G., Peterson, A., ... \& Wilhelm, S. (2008). Managing Tourette syndrome: A behavioral intervention for children and adults therapist guide. Oxford University Press.

Woods, D. W., Piacentini, J. C., Scahill, L., Peterson, A. L., Wilhelm, S., Chang, S., ... \& Rozenman, M. (2011). Behavior therapy for tics in children: acute and long-term effects on psychiatric and psychosocial functioning. Journal of Child Neurology, 26, 858-865. 